ASCLEPIO. Revista de Historia de la Medicina y de la Ciencia

67 (1), enero-junio 2015, p081

ISSN-L:0210-4466

http://dx.doi.org/10.3989/asclepio.2015.07

DOSSIER: CIENCIA Y SABERES AGRÍCOLAS EN CONSTRUCCIÓN EN LA HISTORIA DE AMÉRICA LATINA Y EL CARIBE:

AGENTES, REDES Y CÍRCUITOS (SIGLOS XVIII-XX) / AGRICULTURAL SCIENCE AND KNOWLEDGE IN CONSTRUCTION IN

THE HISTORY OF LATIN AMERICA AND THE CARIBBEAN: NETWORKS, ACTORS AND CIRCUITS (XVIII-XX CENTURIES)

\title{
LA CIENCIA DEL TABACO EN PUERTO RICO, 1900-1940
}

\author{
Teresita Levy, Ph.D. \\ Department of Latin American, Latino and Puerto Rican Studies \\ Lehman College \\ teresita.levy@lehman.cuny.edu
}

Recibido: 12 septiembre 2014; Aceptado: 1 febrero 2015.

\begin{abstract}
Cómo citar este artículo/Citation: Levy, Teresita (2015), "La ciencia del tabaco en Puerto Rico, 1900-1940", Asclepio 67 (1): p081. doi: http://dx.doi.org/10.3989/asclepio.2015.07

RESUMEN: El tabaco se convirtió en un importante producto de exportación para Puerto Rico después de la ocupación norteamericana de 1898, debido a las inversiones en su estudio científico: el cultivo eficiente, la estructura económica y el mercadeo. La experimentación científica y la aplicación práctica de los descubrimientos en las fincas tabacaleras ofrecieron un campo de saber para establecer relaciones cooperativas significativas entre los cultivadores, los científicos en Puerto Rico y EEUU, y el gobierno insular y federal. El compromiso de los puertorriqueños en la ciencia del tabaco, incluyendo su apoyo por la investigación, las demandas continuas para fondos y una legislación agrícola beneficiosa, así como la adopción de la tecnología agrícola moderna, demuestra que éstos no eran pasivos frente a los rápidos cambios económicos de entonces. Por el contrario, ellos eran activistas políticos, usando las garantías democráticas disponibles dentro de la estructura colonial de los EEUU para mejorar su situación económica.
\end{abstract}

PALABRAS CLAVE: Puerto Rico; Tabaco; Experimentación científica; Participación política; Colonialismo.

\section{THE SCIENCE OF TOBACCO IN PORTO RICO, 1900-1940}

ABSTRACT: Tobacco became an important export product in Puerto Rico after the American occupation of 1898, in large part, because of investments in the scientific study of tobacco: its efficient cultivation, its economic structure and its marketing. Scientific experimentation and the practical application of research findings in tobacco farms provided an arena for significant collaborative relationships between tobacco growers, scientists in Puerto Rico and the U.S., and the insular and federal governments. The involvement of Puerto Ricans in the science of tobacco, including their support of scientific inquiry, their continued demands for funding and beneficial agricultural legislation, and their adoption of modern agricultural technology, demonstrate that rather than being observers of rapid economic changes, they were activists, using the democratic guarantees available under the U.S. colonial structure to improve their economic situation.

KEY WORDS: Puerto Rico; Tobacco; Scientific experimentation; Political participation; Colonialism.

Copyright: () 2015 CSIC. Este es un artículo de acceso abierto distribuido bajo los términos de la licencia Creative Commons Attribution-Non Commercial (by-nc) Spain 3.0. 
La investigación y experimentación científica tuvieron un profundo impacto en el desarrollo de la agricultura en Puerto Rico a comienzos del siglo XX. La hoja de tabaco, entre otros productos agrícolas, fue incluido en la estructura tarifaria colonial de los Estados Unidos como un producto libre de arancel. Como resultado de esta ventajosa oportunidad de participar en el mercado estadounidense, los agricultores en las regiones montañosas de la isla convirtieron el cultivo del tabaco en la actividad económica más importante entre 1900 y 1940. Empezando en 1900 hasta 1929, el cultivo del tabaco se expandió rápidamente y consistentemente. Entre 1907 y 1917, el tabaco fue el tercer producto comercial más importante en la isla, después de la caña de azúcar y el café. En 1918, el tabaco sobrepasó al café, convirtiéndose en el segundo producto más importante, posición que ocupó hasta 1940. El valor de exportación del tabaco ascendió en 1920, cuando representó el 38\% del valor de todos los productos comerciales agrícolas, comparado con productos de la caña, los cuales representaron el $25 \%$ ese mismo año (Menéndez Ramos, 1933 y 1934). El cultivo del tabaco no era solo importante para el comercio, sino que servía también como una crucial fuente de ingresos para la población rural. En 1910, más del $14 \%$ de las fincas en Puerto Rico reportaban el cultivo del tabaco, y para 1940, ese número había aumentado al 30\% (Bureau of the Census, 1942d, p. 181). Adicionalmente, la venta de la hoja y la manufactura de los productos tabacaleros eran esenciales para los ingresos del gobierno insular, proporcionando hasta el $30 \%$ de los ingresos del estado durante los años 1930 (Esteva, 1942b, p.7). En definitiva, la importancia del tabaco como producto para el mercado estadounidense, para el bienestar de la población rural, y como fuente de ingresos para el estado insular aumentó espectacularmente entre 1900 y 1940.

Este artículo argumenta que el desarrollo del tabaco como producto de exportación rentable ocurrió, en parte, porque los científicos, los agricultores tabacaleros y el gobierno insular y federal apoyaron la ciencia del tabaco, desde la investigación hasta la aplicación práctica de los descubrimientos científicos. Los funcionarios del gobierno de Puerto Rico, tanto puertorriqueño como norteamericano, creían que el principal obstáculo para la expansión continuada del sector agrícola era la falta de sofisticación en las técnicas agrícolas. Por tanto, instauraron un plan de desarrollo rural basado en la aplicación racional y práctica del conocimiento científico. Los científicos puertorriqueños y americanos, quienes ya estaban enfocados en los problemas particulares del cultivo de la caña, comenzaron un estudio agronómico detallado de la planta del tabaco, incluyendo su ciclo de vida, plagas y enfermedades, para determinar las variedades adecuadas para el terreno de Puerto Rico y los mejores métodos de cultivo.
El gobierno insular tenía que difundir dicha información; después de todo, los descubrimientos científicos solo serían útiles si éstos resultaran en cambios prácticos en los métodos de cultivo. Por ende, las autoridades insulares en Puerto Rico, con la asistencia del gobierno federal de Estados Unidos, establecieron el Departamento de Agricultura y Trabajo y financiaron el desarrollo de estaciones de experimentación agrícola. Estas instituciones eran las responsables tanto de la investigación en el laboratorio como de la aplicación práctica de la tecnología agrícola en las fincas, y crearon un programa de instrucción para agricultores que consistía en demonstraciones en fincas experimentales, visitas a fincas individuales y diseminación en medios de prensa (incluyendo medios impresos y la radio) para acercar la investigación a los campos de cultivo.

Los agricultores del tabaco, al igual que otros cultivadores en la isla, participaron en un proceso de intercambio científico, asistiendo a las presentaciones y demostraciones en las que aprendían nuevas técnicas agrícolas, aplicando estas técnicas en sus fincas y cabildeando ante la legislatura insular para obtener financiación para la investigación relativa al tabaco. Los legisladores insulares, ante la insistencia de los agricultores del tabaco y otros miembros de la Asociación de Agricultores Puertorriqueños, presionaron al Congreso de los Estados Unidos para incluir a Puerto Rico en las leyes que apoyarían la ciencia agrícola ${ }^{1}$. Cuando las agencias del gobierno federal no podían cumplir con las necesidades de investigación del sector tabacalero, los cultivadores presionaron a la legislatura insular para establecer instituciones independientes dedicadas a la investigación del tabaco. La Comisión Para Proteger el Tabaco de Puerto Rico y el Instituto de Tabaco, ejemplos de dichas instituciones, se establecieron con el apoyo financiero del gobierno insular y la cooperación de las comunidades científicas federales e insulares.

Los grupos científicos en Puerto Rico y Estados Unidos también estaban interesados en mejorar las condiciones de vida en las áreas rurales de la isla, donde la gente vivía en la "pobreza y enfermedades atroces» (Clark, 1930, p. 38). La producción agrícola aliviaría, según los funcionarios de gobierno, el desempleo crónico en el campo al emplear familias en las fincas y en el proceso manufacturero. Además, el aumento del cultivo de frutas y vegetales contribuiría al bienestar de las familias, no solo por proveer la nutrición necesaria para una vida saludable, sino que también por ser una fuente posible de ingreso familiar. Los científicos, con el apoyo de los funcionarios del gobierno federal e insular, reconocían que el progreso agrícola requeriría una inversión en el estudio de los métodos de explotación agrícola avanzados y en los temas socioeconómicos que afectaban la vida diaria de los agricultores. Por consiguiente, la ciencia del tabaco se 
amplió para incluir tanto los estudios económicos que investigaban el acceso al crédito, la propiedad de la tierra, y las cooperativas de mercadeo, así como los estudios sociales que trataban temas sobre las condiciones de vida rural y la nutrición adecuada.

La ciencia del tabaco ofreció un campo para establecer relaciones cooperativas entre los agricultores, los científicos y el gobierno insular y federal. El desarrollo y la aplicación práctica de la ciencia del tabaco demuestran que, mucho más que observadores de un cambio económico rápido, como el del sector tabacalero, los puertorriqueños estaban aprovechando los programas federales e insulares que mejorarían su situación económica. La ciencia del tabaco ayudó a los tabacaleros a cultivar de manera eficiente, asegurar un mayor rendimiento por cuerda, y a proveer sustento para sus familias.

\section{EL COMIENZO DE LA INVESTIGACIÓN CIENTÍFICA}

El interés en la investigación científica no era algo nuevo a comienzos del siglo XX, ni era un fenómeno del sistema colonial estadounidense establecido en la isla después de 1898. Las últimas tres décadas del siglo XIX se caracterizaron por un interés sin precedentes en el estudio científico (Becerra de Weierich, 1969, p. 29). Durante este período, la Corona española canceló o redujo los impuestos de importación aplicados a la maquinaria agrícola. Además, comenzaron a circular folletos instructivos por toda la isla, en los cuales se describían nuevas técnicas agrícolas.

Este interés, combinado con las nuevas oportunidades brindadas por la disminución de las restricciones de la Corona española, culminaron en 1888 con el establecimiento de dos estaciones agrícolas (una en Bayamón y la otra en Mayagüez) dedicadas al estudio de las plantas, los insectos, la tierra y el agua, el entrenamiento de capataces para trabajar en las fincas, y la distribución de máquinas para el cultivo. Las dos estaciones también tenían a cargo el estudio del tabaco para mejorar la calidad de las cosechas (Clark, 1930, pp. 47, 102). Desafortunadamente, la falta de fondos forzó el cierre de las estaciones en 1897 y todos los materiales y enseres se vendieron en una subasta pública en 1898 (Clark, 1930, pp. 161-167 y McCook, 2001, p. 54). Estos cierres no invalidaron el interés de los puertorriqueños en utilizar la ciencia para mejorar la agricultura. Por ende, cuando la inversión estadounidense en la agricultura puertorriqueña se tradujo en nuevas posibilidades para la investigación científica, los agricultores puertorriqueños estaban entusiasmados por participar en ellas.

El Departamento de Agricultura de Estados Unidos estableció rápidamente una estación agrícola en Mayagüez y los científicos que trabajaban allí estaban principalmente interesados en los experimentos di- señados para encontrar nuevos productos agrícolas apropiados al clima y la topografía de Puerto Rico. La diversificación del sector agrícola de Puerto Rico no era de interés para los cultivadores de caña ya establecidos, quienes procuraban investigaciones científicas especializadas en la caña (McCook, 2001, p. 55). Así, la Porto Rico Sugar Growers Association fundó la Estación Experimental de Río Piedras en 1910, una institución de investigación científica que se dedicaría solamente a temas del cultivo de la caña (Díaz de Acín, 1967, pp. 165-188). En este momento, la Estación contaba con un personal de seis científicos y un edificio para laboratorios y oficinas (Lugo y Arroyo Aguilú, 2001, p. 21).

Si bien la estación continuaba enfocada en la caña, los científicos estaban bajo la presión de otros agricultores, quienes querían investigar cultivos diversos como, por ejemplo, el tabaco, los cítricos y el café. De hecho, J.T. Crowley, el director de la estación durante sus años iniciales, indicó que muchos agricultores querían establecer subestaciones dedicadas a la investigación de otros cultivos fuera de la caña y señaló el «cordial espíritu de cooperación» que encontró entre ellos (Crowley, 1911, p. 6). Para esta fecha, aunque el cultivo del tabaco se había expandido a casi todas las municipalidades de Puerto Rico, era en las municipalidades de las zonas montañosas en el este de la isla donde se cultivaba el $65 \%$ de la cosecha anual ${ }^{2}$. Precisamente, debido a que la estación estaba en una tierra limítrofe con Caguas, la municipalidad de mayor cultivo de tabaco, el proyecto de investigación del tabaco fue el segundo en establecerse en la Estación y el estudio sistemático de los problemas del tabaco comenzó en $1912^{3}$

En 1914, la Junta de Comisionados de Agricultura, el organismo responsable por la Estación Experimental, decidió que la institución no debía ser propiedad exclusiva de los agricultores dedicados a la caña y que debería ser una organización del estado insular. De este modo, la estación se donó al gobierno insular y la transferencia fue aprobada por la legislatura puertorriqueña el 28 de marzo de 1914. Se cambió el nombre de la estación a Estación Experimental Insular (EEI), un cambio que reflejaba el mandato de que «tiene que haber una ampliación de las actividades de la estación para incluir otros cultivos puertorriqueños» (Board of Commissioners of Agriculture, 1915b, p.9).

\section{LA ESTACIÓN EXPERIMENTAL Y LA INVESTIGACIÓN DEL TABACO}

La investigación sobre el cultivo del tabaco se expandió inmediatamente y se registró un aumento en el número de científicos dedicados al estudio de todos los aspectos del sector tabacalero, desde la plantación de semilleros hasta el proceso de manufactura. John 
A. Stevenson, un patólogo del tabaco, estudió la enfermedad del moho de los puros que tendía a aparecer varios días después de la manufactura (Board of Commissioners of Agriculture, 1915b, p.41). R. T. Cotton estudió el ciclo de vida de los escarabajos, la pulga del tabaco, considerada «el peor enemigo del tabaco» (Board of Commissioners of Agriculture, 1916c, p.86). Un año después, Cotton estaba a cargo de la plantación de un lote experimental de tabaco en las tierras de la Estación Experimental Insular (Insular Experiment Station of Puerto Rico, 1917, p. 107). En 1917, los científicos del tabaco estaban ocupados estudiando las lapas, y detallaron un plan de acción para tratar el problema (Insular Experiment Station of Puerto Rico, Department of Agriculture and Labor of Puerto Rico, 1919a, p. 128). En breve, el tabaco se convirtió en un proyecto tan central para la EEI como la investigación de la caña de azúcar, lo que refleja el aumento de la importancia del tabaco como producto comercial para la exportación.

La Ley Orgánica del 2 de marzo de 1917 creó el Departamento de Agricultura y Trabajo (DAT), colocando la responsabilidad del bienestar del sector agrícola en manos del estado insular (Camuñas, 1917a, p. 545). Uno de los principales objetivos del DAT era «aumentar y mejorar la producción, mejorar las prácticas [agrícolas] existentes y presentar otras nuevas que contribuyeran al desarrollo económico de la isla» (Camuñas, 1917a, p. 545). Para cumplir con estos objetivos, la Resolución Conjunta no.18 firmada en noviembre de 1917 formalizó la ya fuerte relación entre el DAT y la Estación Experimental Insular al transferir la jurisdicción de la Estación al DAT (Díaz de Acín, 1967, p. 8).

La transferencia tenía sentido a nivel administrativo, ya que existía una gran cooperación entre los científicos de la EEI y los del Departamento de Agricultura de los Estados Unidos. La cooperación era continua y los científicos americanos viajaban con frecuencia a las estaciones agrícolas de la isla para adiestramientos, demostraciones y experimentos. Por ejemplo, E. E. Barker, un científico en el Departamento de Reproducción de Plantas en la Universidad de Cornell, visitó Puerto Rico en 1920 para realizar experimentos de selección de semillas en las zonas tabacaleras (Insular Experiment Station of Puerto Rico, Department of Agriculture and Labor of Puerto Rico, 1921c, p. 32). Los científicos americanos también actuaban como personal de la EEI. Durante el tiempo en el cual George N. Wolcott trabajó como entomólogo de la EEI, por ejemplo, se realizaron los primeros estudios sobre el gusano de candela en 1922 (Insular Experiment Station of Puerto Rico, Department of Agriculture and Labor of Puerto Rico, 1924e, p. 24). Igualmente, los científicos puertorriqueños participaban en todos los aspectos de la investigación del tabaco en la estación como líderes de proyecto, personal auxiliar y en puestos administrativos de alto nivel. J. A. B. Nolla se unió al personal de la EEI a comienzos de 1920 y para 1926 estaba a cargo del laboratorio de las fincas de tabaco (López Domínguez, 1929b, p. 30)4 . Carlos Chardón, quizás el investigador agrícola más importante y quien será eventualmente hacedor de políticas económicas y canciller de la Universidad de Puerto Rico, comenzó su actividad como funcionario público trabajando en experimentos de tabaco en la EEI. Dicha participación en la vida científica de la isla por parte de los científicos tanto americanos como puertorriqueños sugiere que las agencias agrícolas federales estaban tan interesadas en la modernización de las prácticas agrícolas como las insulares, y que los científicos de la isla y de Estados Unidos estaban en constante comunicación.

La colaboración entre el DAT y la EEl fue crucial para la implementación de los descubrimientos científicos en todas las áreas rurales. Ambas instituciones no solo ahondaban en todos los aspectos del estudio científico, sino que estaban también involucradas en la popularización de los «métodos modernos de agricultura». El informe anual de 1920 de la EEI señaló que "lo mínimo que la gente de Puerto Rico puede exigir de su estación experimental es un contacto íntimo y constante con las necesidades y prácticas de agricultura que determinan nuestra producción; la satisfacción de los primeros y la explicación, confirmación o corrección de los segundos, y la sustitución por otros más modernos y convenientes» (Camuñas, 1919c, p. 687). Los científicos afiliados con el DAT y la EEI aceptaron la orden de "contacto íntimo y constante» con seriedad. En 1920, los inspectores agrícolas realizaron 3.761 visitas en toda la isla en las cuales valoraron las condiciones de vida, el número de cuerdas dedicadas a cada cultivo, el promedio cosechado por acre y los precios pagados por los cultivos (Gandía Córdova, 1920, pp. 518-519). Estos inspectores también asesoraban e instruían a los agricultores sobre los últimos métodos de control de plagas y enfermedades, la aplicación más productiva de los abonos y ofrecían ideas sobre la comercialización de sus cultivos efectivamente. La instrucción ocurría de manera individual, o en conferencias impartidas a un grupo por los inspectores agrícolas. De las 228 conferencias impartidas en 1920, un 50\% tuvo lugar en los distritos tabacaleros. Además, casi la mitad de las más de 13.000 personas que asistieron a estas clases, lo hicieron en regiones tabacaleras (Gandía Córdova, 1920, pp. 518-519). Probablemente, los participantes eran agricultores del tabaco interesados en aprender la aplicación de los descubrimientos científicos para mejorar la calidad y la cantidad de sus cultivos. Los agricultores tabacaleros esperaban que un mejor producto contribuyera a un mayor precio en el mercado. 
A partir de 1920, un cambio de actitud se tornó evidente en la comunidad científica y en las agencias gubernamentales que trataban con la agricultura. Los científicos, tanto como los funcionarios del gobierno afiliados con ellos, reconocieron que la investigación agrícola y la implementación práctica de los descubrimientos científicos solo garantizarían un cultivo saludable, y que la verdadera modernización y el desarrollo agrícola sostenido requerirían la participación de otras agencias y la mejora de la infraestructura económica y física de la isla. Los científicos agrícolas, con el apoyo del DAT, comenzaron a presionar en la legislatura insular y federal para obtener fondos adicionales que permitieran realizar estudios de las condiciones económicas y sociales en todo Puerto Rico. El alto costo del transporte de los productos agrícolas desde el interior de la isla hasta las instalaciones de transporte marítimo, el establecimiento de estructuras adecuadas de crédito agrícola, la escasez de madera combustible y materiales para la construcción rural, y la falta de organizaciones para facilitar el mercadeo de los productos agrícolas fueron factores importantes para el éxito de una cosecha tabacalera, tanto como el tipo de semilla sembrada (Insular Experiment Station of Puerto Rico, Department of Agriculture and Labor of Puerto Rico, 1920 b, p. 58). Asimismo, estaba bajo escrutinio el bienestar de la población rural en cuanto a nutrición, salud, e higiene. Esto marcó el comienzo de la expansión de la misión de la $\mathrm{EEI}$, de una organización puramente dedicada a la investigación agrícola a una interesada en la modernización de las áreas rurales y el desarrollo generalizado de la agricultura puertorriqueña.

La década de 1920 fue de crecimiento para la Estación Experimental Insular. F. A. López Domínguez, director de la EEI durante este tiempo, observó que «el interés del público en el trabajo de la Estación y el deseo general despertado en el cultivador inteligente de mejorar los métodos y procedimientos, han creado una demanda de más y más cooperación por parte de la Estación con los cultivadores, e incluso los industriales de la isla, en forma de ayuda directa para la inmediata solución de sus problemas cotidianos» (López Domínguez, 1926a, p. 21). Este interés tuvo como resultado la creación de un programa de educación en el que los científicos visitaban fincas para enseñar a los agricultores nuevas técnicas de cultivo. Estas fincas podían ser privadas, prestadas por sus dueños interesados en aprender nuevos métodos agrícolas, o podían ser tierras administradas por la EEI y designadas como "fincas de demostración.» Debido al reto que constituía difundir la información a los agricultores principalmente analfabetos, las visitas a las fincas eran la manera más eficaz para la difusión de conocimientos científicos. Para los agricultores de tabaco, las fincas de demostración constituían una oportunidad de ver por sí mismos el éxito de los métodos agrícolas modernos, tales como la protección de los semilleros con tela, el uso de cantidades apropiadas de abonos, el curado adecuado de la hoja y los métodos de almacenamiento. Aquellos que no podían viajar a las fincas de demostración aprovechaban las visitas a las fincas privadas por parte de los científicos de la EEI y de los especialistas del Departamento de Agricultura. Durante estas visitas, los científicos y agentes del DAT demostraban nuevas técnicas de siembra, respondían a preguntas sobre los programas del gobierno para la agricultura, y hablaban sobre las condiciones del mercado para cultivos particulares. La participación de los agricultores en dichos programas era constante y demostraba la voluntad de experimentar con métodos mejores de cultivo y técnicas científicas modernas que podrían ofrecerles mejores ganancias. El informe de 1929-1930 de la EEI señaló que «los cultivadores han aprovechado más las oportunidades de servicio ofrecidas por la Estación» (Insular Experiment Station of Puerto Rico, Department of Agriculture and Labor of Puerto Rico, 1931g, p. 5).

Una breve discusión de los cambios en el rendimiento promedio de la hoja de tabaco por cuerda en las regiones tabacaleras desde 1910 hasta 1940, ilustra el impacto de los programas de la EEI en el cultivo del tabaco. Los especialistas del tabaco de la EEI habían señalado la disminución de los minerales del suelo después de una cosecha de tabaco y la mala calidad de la semilla de tabaco en toda la isla como dos problemas inmediatos a resolver. Los experimentos con diferentes tipos de fertilizantes, así como los experimentos en la reproducción de plantas comenzaron en 1916 y continuaron hasta 1938 (Cotton, 1917, p. 107). El objetivo era aumentar el rendimiento por cuerda hasta un promedio de 800 libras para que se pudiera reducir el número de cuerdas utilizadas para el tabaco. Esas tierras disponibles se podrían destinar a otros cultivos tales como los vegetales, los tubérculos y las frutas (Agricultural Experiment Station, College of Agriculture and Mechanic Arts, University of Puerto Rico, 1939d, p. 100). Además, «nuevas variedades con mayor capacidad de rendimiento, buena calidad y resistencia a las enfermedades, permitirían al cultivador restringir sus fincas a las tierras más fértiles sin reducir su producción total» (Agricultural Experiment Station, College of Agriculture and Mechanic Arts, University of Puerto Rico, 1939d, p. 46).

Los científicos de la $\mathrm{EEI}$, quienes habían tenido éxito en los experimentos en las fincas de la Estación, compartieron las nuevas técnicas con los agricultores de las regiones tabacaleras. En 1910, el rendimiento promedio por cuerda era de 467 libras de hojas de tabaco. Luego de trece años de experimentación, el informe de la EEI de 1923 indicó que el cultivo de tabaco era más rentable y que la "selección de variedades más productivas, mejores métodos para los semilleros y las fincas, el uso de fertilizantes y la 
adopción de métodos de control de las enfermedades y del daño causado por los insectos han logrado maravillas» (Insular Experiment Station of Puerto Rico, Department of Agriculture and Labor of Puerto Rico, $1924 f$, p. 18). Para 1940 , el rendimiento promedio había aumentado a 675 libras por cuerda, un aumento del 44\% desde 1910 (Bureau of the Census, 1913a, pp. 72-77; 1942d, pp. 226, 237). La mejora en el rendimiento se debía a los cambios en el uso de abonos, semillas u otros métodos generales de cultivo. Además, un $81 \%$ de todas las municipalidades en donde se cultivaba tabaco aumentaron su rendimiento por cuerda entre 1910 hasta 1940, un logro destacable que evidencia la efectividad de los programas de la EEI. Este aumento generalizado del rendimiento por cuerda indicaba que no eran solo dos o tres fincas enormes las cuales adoptaron métodos científicos agrícolas, sino que de hecho también un gran número de fincas de pequeña escala - la mayoría de las fincas tabacaleras medían menos de 10 cuerdas - los adoptaron también.

\section{LA EXPANSIÓN DE LA EDUCACIÓN CIENTÍFICA Y EL SERVICIO DE EXTENSIÓN AGRÍCOLA}

El éxito de los programas para aumentar el rendimiento de hoja por cuerda demostró a los científicos que las visitas de los agentes a las fincas eran el mejor método de instrucción científica. La expansión de dichos programas de educación dependía del presupuesto asignado para la Estación Experimental, el cual se había tornado problemático desde su traslado al gobierno insular en 1914. Hasta 1917, cuando se colocó a la EEI bajo la jurisdicción del Departamento de Agricultura y Trabajo, los fondos para su operación provenían de la tesorería insular, aunque algunos experimentos eran financiados por el Departamento de Agricultura de Estados Unidos y sus afiliados. Hubo muchos intentos de hacer frente a la falta de un presupuesto estable para las operaciones mediante el establecimiento de impuestos sobre materiales para la explotación agrícola, tales como los alimentos para el ganado y los fertilizantes, pero la estabilidad no se conseguía y el presupuesto variaba anualmente. La ayuda permanente llegó en 1931, cuando la Ley Smith-Lever de 1914 se extendió a Puerto Rico gracias al continuo cabildeo de la Asociación de Agricultores Puertorriqueños. Esta ley federal asignó fondos para la divulgación de los programas de investigación agrícola realizados en universidades a través del Servicio de Extensión Agrícola. La ley se aprobó en la legislatura puertorriqueña en 1934, y en ese momento el Departamento de Agricultura insular transfirió la jurisdicción de la EEI al Colegio de Ciencias Agrícolas de la Universidad de Puerto Rico en Mayagüez. El cambio de jurisdicción significaba que la EEI podía aprovechar los fondos garantizados por la legislación Hatch-Adams y Smith-Lever y que por primera vez, la EEI de hecho tendría un presupuesto operativo independiente de los fondos reunidos por la imposición tributaria

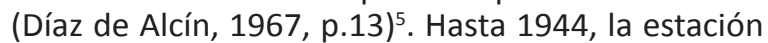
recibiría un presupuesto de hasta $\$ 25,000$, y luego un aumento anual de $\$ 5,000$ en virtud de la ley (Agricultural Experiment Station, College of Agriculture and Mechanic Arts, University of Puerto Rico, 1935a, p. 6). Se cambió nuevamente el nombre de la EEI a Estación Experimental Agrícola del Colegio de Agricultura y Artes Mecánicas de la Universidad de Puerto Rico (EEA).

Los fondos federales permitieron a la Estación Experimental Agrícola, en colaboración con el Servicio de Extensión Agrícola, hacer frente finalmente a la declaración sobre ampliar la misión, desarrollada en la década de 1920 y estudiar "los muchos problemas económicos que afectan el bienestar de los cultivadores y la industria agrícola de Puerto Rico» (Cook y Otero, 1937, p. 77). Se creó una División de Economía Agrícola para investigar estos problemas. Los estudios comenzaron inmediatamente e incluyeron la disponibilidad de créditos e hipotecas, recibos y gastos de las pequeñas granjas, y la eficiencia de la mano de obra de la granja. Estos estudios contribuyeron a la comprensión de los problemas socioeconómicos que afectaban a los cultivadores del tabaco y también al sector agrícola en su totalidad. Ello reflejó un interés de la comunidad científica de la isla y el continente en mejorar las condiciones de vida de los puertorriqueños en las áreas rurales.

El Servicio de Extensión Agrícola experimentó un período de próspera actividad desde 1936 hasta 1940, ya que fueron los Agentes de Extensión Agrícola, tales como agrónomos profesionales, los que estuvieron encargados de divulgar las nuevas investigaciones de la Estación Experimental Agrícola. Cada agente fue asignado a un distrito en particular, constituido de varias municipalidades. Una vez en su distrito, los agentes organizaron Juntas de Cultivadores que ayudarían a los agentes para formular un plan de educación científica ajustado a un área en particular. En las regiones tabacaleras, por ejemplo, los agentes del Servicio de Extensión asistían a los agricultores con la aplicación de fertilizantes, la preparación de semilleros, el manejo de las plagas y las enfermedades, y el mercadeo de su producto (Servicio de Extensión Agrícola, Colegio de Agricultura y Artes Mecánicas de la Universidad de Puerto Rico, 1938, pp. 7-8).

El primer informe oficial del Servicio de Extensión Agrícola, comentaba que «un $50 \%$ de todas la granjas recibieron la visita de los Agentes de Extensión Agrícola... [como parte de] los programas de educación y servicio para mejorar las condiciones sociales y eco- 
nómicas de las zonas rurales» (Servicio de Extensión Agrícola, Colegio de Agricultura y Artes Mecánicas de la Universidad de Puerto Rico, 1938, p. 5). Los agentes realizaron demostraciones de técnicas agrícolas, explicaron a los agricultores la continuamente alterada legislación federal o insular para que pudieran aprovechar los programas agrícolas, y hablaron sobre las preocupaciones ambientales, tales como la conservación de los suelos, la erosión del terreno, y la calidad del agua. El número de visitas realizadas por Agentes de
Extensión Agrícola, al igual que el número de hogares individuales y granjas, se triplicó entre 1936 y 1940. (Ver Tabla I). Cientos de miles de hombres, mujeres y niños participaron en los Programas de servicio de extensión, no solo en las demostraciones en las fincas, sino que también visitando una oficina del Servicio de Extensión Agrícola, ofreciéndose como líderes comunitarios para implementar programas o sumándose a los Clubs 4-H, los programas específicos para la juventud del Servicio de Extensión Agrícola.

Tabla I. Visitas y demostraciones de agentes de extensión agrícola, 1936-1940

\begin{tabular}{|l|l|l|l|l|l|}
\hline & 1936 & 1937 & 1938 & 1939 & 1940 \\
\hline Número total de visitas a hogares y granjas & 27.894 & 36.459 & 54.993 & 80.758 & 88.571 \\
\hline Número de hogares y granjas distritales visitadas & 13.233 & 16.573 & 26.312 & 37.886 & 43.208 \\
\hline Número total de demostraciones & 322 & 2.173 & 2.812 & 3.806 & 4.820 \\
\hline Número total de asistentes & $\mathrm{n} / \mathrm{d}$ & 29.144 & 34.445 & 39.689 & 54.590 \\
\hline
\end{tabular}

Fuente: A. Rodríguez Géigel, Sirviendo al agro. Informe anual del Servicio de Extensión Agricola. Colegio de Agricultura y Artes Mecánicas Universidad de Puerto Rico. Diciembre 1, 1939 a noviembre 30, 1940, Río Piedras, PR, Universidad de Puerto Rico, 1940, pp. 8-9.

Desafortunadamente, no es posible distinguir las visitas que se realizaron únicamente a hogares y cuáles fueron a las fincas en tierras cultivadas. Debido a que el Servicio de Extensión Agrícola defendía que la finca estaba intrínsecamente unida al hogar, los datos sirven para observar el nivel de actividades de educación científica en las zonas rurales. Los datos sugieren que el contacto entre los científicos y el público era verdaderamente constante y cercano.

El declive de los precios del tabaco en la década de 1930 y las dificultades generalizadas enfrentadas por los puertorriqueños de las zonas rurales durante la Gran Depresión, tuvieron como resultado la concentración de la investigación científica en los cultivos de subsistencia. Al mismo tiempo, los fondos apropiados para la investigación específica del tabaco comenzaron a disminuir. El interés científico, como se señaló antes, se había extendido a otras áreas socioeconómicas y el dinero se desvió a otras industrias agrícolas, tales como el ganado, las aves y los lácteos. Para 1940, la mayoría del trabajo realizado por la EEA y el Servicio de Extensión Agrícola se limitaba a los estudios socioeconómicos o proyectos para aumentar el cultivo de subsistencia (Agricultural Experiment Station, College of Agriculture and Mechanic Arts, University of Puerto Rico, 1941f, p. 8). En 1941, ante la posibilidad inminente de que los Estados Unidos se involucraran en el conflicto armado en Europa, se ordenó al Servicio de Extensión Agrícola «el desarrollo de una intensa campaña en toda la isla en la que se demostrara a los cultivadores la necesidad de plantar productos de subsistencia a mayor escala para el uso en su granja y también para satisfacer las exigencias del mercado local» (Rodríguez Géigel, 1941, p. 3).

El enfoque en la investigación y la educación relacionadas con la subsistencia redujo dramáticamente los fondos para la investigación del tabaco. A fin de mejorar la situación, los agricultores tabacaleros, con el apoyo de la Asociación de Agricultores, presionaron incesantemente ante el gobierno para proteger al sector tabacalero, realizaron campañas de prensa negativas que mostraban a los funcionarios del gobierno como abusivos e indiferentes ante la grave situación de los cultivadores y se unieron a organizaciones agrícolas que sabían cómo presionar y manipular las burocracias insulares y federales ${ }^{6}$. El resultado de tanta presión fue el establecimiento de instituciones dedicadas, entre otros objetivos, a la continuación de la investigación científica en el tabaco.

\section{LA COMISIÓN PARA PROTEGER EL TABACO Y EL INSTITUTO DEL TABACO}

La legislatura puertorriqueña estableció la Comisión Para Proteger el Tabaco de Puerto Rico el 18 de febrero de 1929, bajo la supervisión de una junta ejecutiva constituida por «cultivadores de tabaco de buena fe» (Comisión Para Proteger el Tabaco de Puerto Rico, 1930, p. 3). La Comisión estaba comprometida a apoyar cualquier esfuerzo para que el sector tabacalero siguiera vigente como fuente de ingresos para los agricultores del área montañosa. El primer punto de la agenda 
de la Comisión era establecer juntas locales en cada municipalidad en la que se cultivaba tabaco (Comisión Para Proteger el Tabaco de Puerto Rico, 1930, p. 13). Los cultivadores de tabaco participaron activamente en el establecimiento de estas juntas locales y tan solo un año después de la constitución de esta Comisión, operaban juntas locales en todos los distritos tabacaleros. Las juntas asistían a la Comisión para brindar información sobre las condiciones de los cultivos durante el período de la cosecha, la cantidad de cuerdas cultivadas y el rendimiento esperado por cuerda; información que la Comisión entonces publicaba en Circulares y distribuía a través de la isla. La Comisión también debía llevar a cabo una «encuesta del problema del mercado del tabaco en Puerto Rico, estudiando los métodos de cultivo y la administración agrícola, y realizando todos los estudios conducentes o necesarios para proteger y mejorar las condiciones agroindustriales del tabaco puertorriqueño» (Comisión Para Proteger el Tabaco de Puerto Rico, 1930, pp. 16-18). Una parte importante de la encuesta se volcaría en un censo tabacalero detallado en el que se incluirían los nombres de los cultivadores de tabaco, los acres plantados, los abonos usados y las cantidades, el número de fincas de curación, el rendimiento promedio por cuerda y el costo promedio de una cosecha.

Con la ayuda de los especialistas en tabaco de la Estación Experimental Agrícola, la Comisión organizó fincas experimentales para realizar pruebas de producción comparativa de variedades de tabaco que eran más resistentes a las enfermedades y de mejor gusto en general. Al igual que la EEA, los objetivos de la Comisión eran más amplios que el descubrimiento científico e incluían un estudio del desarrollo y la administración de las cooperativas de tabaco que existían en los Estados Unidos, así como un continuo examen de la situación del mercado mundial para el tabaco puertorriqueño.

Debido a que el presupuesto operativo de la Comisión dependía de las contribuciones de los agricultores tabacaleros, la Comisión se convirtió en una carga económica para los cultivadores. El ingreso de una cosecha de tabaco era apenas suficiente para pagar a los jornaleros y cubrir el contrato de refacción. Pedir a los pequeños productores que donasen los pocos recursos monetarios que tenían era un problema. La Comisión influía continuamente ante la legislatura insular para obtener aumentos en la asignación de fondos para la investigación del tabaco, argumentando que era injusto cargar a los individuos con la financiación de la investigación cuando esto era el deber del estado insular. La Comisión pervivió hasta 1936, pero con fondos tan limitados que su trabajo se vio restringido a informes semanales sobre el estado de la industria y a la cooperación con esfuerzos legislativos dirigidos por la Asociación de Agricultores Puertorriqueños.
Los agricultores tabacaleros, decepcionados con el destino de la Comisión, solicitaron nuevamente el apoyo de la Asociación de Agricultores Puertorriqueños. Los cultivadores reconocían la importancia de continuar las investigaciones científicas sobre los problemas del tabaco y, junto con la Asociación, presionaron a la legislatura puertorriqueña para establecer una institución cuyo único propósito era patrocinar la investigación que aliviaría los problemas económicos y agrícolas de los agricultores tabacaleros. El Instituto de Tabaco de Puerto Rico se constituyó a través de la Resolución Conjunta no. 15 de la legislatura puertorriqueña el 22 de julio de 1935, como agencia del gobierno bajo el liderazgo de una junta ejecutiva constituida por el Comisionado de Agricultura y Comercio, y dos cultivadores de tabaco y dos industriales del tabaco designados por el Gobernador de Puerto Rico, bajo el consentimiento del estado insular (Nolla, 1937, p. 7). A diferencia de la Comisión, que dependía de las contribuciones de los cultivadores de tabaco, el Instituto obtendría su presupuesto de una apropiación insular. El dinero adicional provendría de un impuesto de 15 centavos por cada quintal de tabaco vendido en la isla (Esteva, 1940a, p. 5).

El Instituto tenía tanto objetivos agrícolas como industriales. Su misión agrícola era encontrar una «solución a los problemas relativos al cultivo de tabaco» y "la manipulación del cultivo después de la cosecha». Para hacer esto, el Instituto estableció ramas de investigación en agronomía, química, patología y genética. Por su parte, la misión industrial era investigar «los posibles mercados y nuevos usos para el tabaco». El Instituto también era responsable por la promoción de «legislación que beneficiara a la industria del tabaco en su totalidad» (Esteva, 1940a, p. 5).

La Estación Experimental Agrícola colaboró con el Instituto desde sus inicios. En 1935, la Universidad de Puerto Rico cedió 12 cuerdas de tierra al Instituto para establecer una planta física. Una nueva construcción, financiada con dinero de la Administración Federal de Asistencia para Emergencias, alojaba la oficina del Director, una sala de conferencias y laboratorios de química, genética y patología (Nolla, 1937, p. 36) ${ }^{7}$. Al igual que la EEA, el Instituto estaba comprometido a la amplia difusión de los descubrimientos científicos. Para cumplir con este compromiso, el Instituto instauró una biblioteca con copias de publicaciones sobre agricultura que trataban el tema del cultivo de tabaco, tales como la revista Tobacco y la Revista de Agricultura de Puerto Rico, así como artículos académicos presentados en conferencias locales e internacionales (Esteva, 1940a, pp. 26-27). 
El Instituto se convirtió en la voz de la industria tabacalera en la legislatura. Debido a la preocupación sobre la autenticidad del tabaco que se vendía en Estados Unidos, bajo la etiqueta de «Tobacco from Puerto Rico» (Tabaco de Puerto Rico), el Instituto presentó con éxito una legislación que regulaba dicha etiquetación. La ley no. 16 se promulgó el 10 de junio de 1939 y estableció que solo el tabaco que en realidad se hubiera cultivado en Puerto Rico podría etiquetarse como tal (Esteva Jr., 1940a, p. 28). Algunos de los esfuerzos legislativos del Instituto no tuvieron éxito. Por ejemplo, el Instituto patrocinó el proyecto de ley no. 718 para establecer un sistema de clasificación uniforme para el tabaco para cigarrillos. El propósito de la ley era proteger a los agricultores de la clasificación arbitraria de sus cultivos por parte de los distribuidores, compañías tabacaleras y financieros. Aunque el proyecto de ley contaba con el fuerte apoyo de los agricultores, se enfrentó a una fuerte oposición por parte de los compradores de la hoja y nunca llegó a debatirse en la legislatura.

La capacidad del Instituto de lograr el cambio se vio disminuida por el continuo declive del sector del tabaco. Aunque tanto la Comisión como el Instituto tuvieron éxito en continuar la investigación científica enfocada en el tabaco, el sector agrícola (y los fondos para el desarrollo y apoyo del sector) pronto entrarían en un período de declive permanente. El colapso económico en la década del 1930, cambios en preferencias del consumidor a fumar cigarrillos en vez de cigarros, la destrucción de cosechas por huracanes y tormentas, y el interés del gobierno insular en buscar alternativas industriales para el desarrollo económico de la isla contribuyeron a que el sector tabacalero careciera del apoyo insular y federal para la continuación de los programas económicos y científicos que lo protegían.

\section{CONCLUSIÓN}

La difusión del conocimiento científico en las áreas rurales de Puerto Rico fue un proyecto colaborativo entre la comunidad científica, los agricultores puertorriqueños y el gobierno insular y federal. La aplicación práctica de los descubrimientos de investigación científica en las fincas tuvo un profundo impacto en las áreas rurales de Puerto Rico entre 1910 y 1940. En las regiones tabacaleras de Puerto Rico, los científicos demostraron que la selección de variedades más productivas de tabaco, el mantenimiento de mejores semilleros, el uso de los abonos adecuados y la adopción de métodos de control de las enfermedades y las plagas de los insectos lograban un cultivo más lucrativo. La ciencia del tabaco mejoró exitosamente la calidad del producto y aumentó el potencial de ganancia de los cultivadores.
Los agricultores tabacaleros adoptaron nuevas técnicas como resultado de estos esfuerzos y se involucraron en un proceso colaborativo de intercambio científico: la Estación suministró nuevas tecnologías agrícolas y los cultivadores prestaron sus fincas para la aplicación práctica de estas tecnologías. Su organización como fuerza política y su participación en el proceso científico fueron cruciales para garantizar que la investigación dedicada al tabaco continuara frente a la disminución de fondos por parte del gobierno insular. Su constante presión a la legislatura insular, con la asistencia de la Asociación de Agricultores Puertorriqueños, se tradujo en el establecimiento de la Comisión Para Proteger el Tabaco de Puerto Rico y del Instituto de Tabaco. La legislatura federal no escapó de la presión política de los cultivadores de tabaco, quienes lograron ganar la extensión de las leyes agrícolas federales que garantizaron los fondos para la investigación científica en Puerto Rico. A través de su participación en la ciencia del tabaco, desde las fincas hasta las salas de la legislatura insular y federal, los cultivadores de tabaco se convirtieron en agricultores modernos, preocupados por la utilización de la tecnología de cultivo más eficiente para aumentar su rentabilidad y, de este modo asegurar el bienestar de sus familias. Más importante aun, el éxito de la ciencia del tabaco demuestra que los legisladores, científicos y agricultores puertorriqueños aprovecharon la nueva relación colonial con los Estados Unidos para acceder a fondos de investigación científica, crear instituciones y participar en programas federales que mejoraban la posibilidad de cultivar lucrativamente productos para el mercado estadounidense.

La ampliación de los objetivos de la Estación experimental agrícola para incluir la economía del hogar, la administración de la granja y el desarrollo de la infraestructura rural demuestra que había interés no solo en maximizar la eficiencia de los cultivos sino también en la modernización de la sociedad rural, y en consecuencia, Puerto Rico en su totalidad. Estos amplios programas socioeconómicos no eliminaron, sin embargo, la miseria en las áreas rurales de Puerto Rico. La naturaleza volátil de un sector agrícola dependiente de las fluctuaciones internacionales de oferta y demanda, combinado con las continuas reducciones de la financiación para la investigación agrícola y la educación, contribuyeron al impacto limitado de los programas de modernización rural. De todos modos, la participación de los cultivadores en programas científicos demuestra claramente que los puertorriqueños comprendían el papel de la ciencia en la modernización de la isla y que participaron por voluntad propia y con entusiasmo en la experimentación científica y su aplicación práctica en las fincas. 


\section{NOTAS}

1 La Asociación de Agricultores Puertorriqueños era la organización más grande e importante para los agricultores de Puerto Rico. Fundada en el 1925, la Asociación se dedicó a ser la voz del agricultor en la legislatura insular y federal.

2 Para determinar las municipalidades más importantes en el cultivo del tabaco, es necesario calcular el porcentaje de cuerdas dedicadas al cultivo del tabaco y el número de libras producidas por cada municipalidad, empezando en el 1900 y terminando en el 1940. Las municipalidades tabacaleras en la zona montañosa este eran Aguas Buenas, Aibonito, Barranquitas, Bayamón, Caguas, Cayey, Cidra, Comerío, Corozal, Gurabo, Humacao, Juncos, Naranjito, San Lorenzo, Toa Alta y Yabucoa. Estas municipalidades eran las más importantes en el cultivo del tabaco, no solo porque cultivaban el mayor numero de cuerdas, pero porque producían las más libras de hoja (Bureau of the Census, 1913a, pp. 72-77; 1922b, pp. 408-415; 1932c, pp. 232-237; 1942d, p. 60).

3 Caguas cultivó 1.974.900 libras de tabaco, más de un millón de libras más que Cayey, la siguiente municipalidad más grande de cultivo de tabaco (Bureau of the Census, 1913a, pp. 72-77).

\section{BIBLIOGRAFÍA}

Agricultural Experiment Station, College of Agriculture and Mechanic Arts, University of Puerto Rico (1935a), Annual Report of the Director for the Year 1933-34, San Juan, Bureau of Supplies, Printing, and Transportation.

Agricultural Experiment Station, College of Agriculture and Mechanic Arts, University of Puerto Rico (1937b), Annual Report of the Agricultural Experiment Station. Fiscal Year 1935-36, San Juan, Bureau of Supplies, Printing, and Transportation.

Agricultural Experiment Station, College of Agriculture and Mechanic Arts, University of Puerto Rico (1938c), Annual Report of the Agricultural Experiment Station. Fiscal Year 1936-37, San Juan, Bureau of Supplies, Printing, and Transportation.

Agricultural Experiment Station, College of Agriculture and Mechanic Arts, University of Puerto Rico (1939d), Annual Report of the Agricultural Experiment Station. Fiscal Year 1937-38, San Juan, Bureau of Supplies, Printing, and Transportation.

Agricultural Experiment Station, College of Agriculture and Mechanic Arts, University of Puerto Rico (1939e), Annual Report of the Agricultural Experiment Station. Fiscal Year 1938-39, San Juan, Bureau of Supplies, Printing, and Transportation.

Agricultural Experiment Station, College of Agriculture and Mechanic Arts, University of Puerto Rico (1941f), Annual Report of the Agricultural Experiment Station. Fiscal Year 1940-41, Lancaster Press.

Anónimo (1929), "El Gobernador veda el proyecto del tabaco: Un nuevo aspecto de la acción destructora del Gobernador", El Agricultor Puertorriqueño, 7 (9), p. 26

Anónimo (1934), “Demanda contra la Comisión Protectora del Tabaco declarada sin lugar", El Agricultor Puertorriqueño, 14 (6), p. 15.

Becerra de Weierich, Isabel (1969), Las primeras estaciones agronómicas en Puerto Rico, Master's, tesis. University of Puerto Rico.
4 Nolla luego se desempeñó como Director del Instituto de Tabaco.

5 La ley Hatch de 1887 autorizó el uso de fondos federales para el establecimiento de estaciones experimentales de agricultura en conexión con el colegio.

6 Ver como ejemplos (1929), «El Gobernador veda el proyecto del tabaco: Un nuevo aspecto de la acción destructora del Gobernador», El Agricultor Puertorriqueño, 7 (9), p. 26; (1934), «Demanda contra la Comisión Protectora del Tabaco declarada sin lugar», El Agricultor Puertorriqueño, 14 (6), p. 15.

7 La Administración Federal de Asistencia para Emergencias (FERA pos sus siclas en inglés) brindaba asistencia a los trabajadores desempleados a través de la financiación de proyectos de obras públicas. Debido a que la Universidad de Puerto Rico y el Instituto de Tabaco eran instituciones públicas en tierra pública, los fondos de la FERA se podían usar para construir instalaciones tales como laboratorios, invernaderos u otros edificios. Descripciones de las facilidades se encuentran también en Esteva, 1940a, pp. $12-14$

Board of Commissioners of Agriculture (1913a), Second Report of the Board of Commissioners of Agriculture for the Period from January 1, 1912 to June 30, 1913. Issued June 30, 1913, San Juan Bureau of Supplies, Printing, and Transportation.

Board of Commissioners of Agriculture (1915b), Third Report of the Board of Commissioners of Agriculture for the Period from July 1, 1913 to July 1, 1914, San Juan, Bureau of Supplies, Printing, and Transportation.

Board of Commissioners of Agriculture (1916c), Fourth Report of the Board of Commissioners of Agriculture for the Period from July 1, 1914 to July 1, 1915, San Juan, Bureau of Supplies, Printing, and Transportation.

Board of Commissioners of Agriculture (1917d), Fifth Report of the Board of Commissioners of Agriculture for the Period from July 1, 1915 to June 30, 1916. Issued June 30, 1916, San Juan, Bureau of Supplies, Printing, and Transportation.

Bureau of the Census (1913a), Thirteenth Census of the United States Taken in the Year 1910. Statistics for Porto Rico: Agriculture, Washington, DC.

Bureau of the Census (1922b), Fourteenth Census of the United States Taken in the Year 1920, Vol. 6, Pt. 3, Agriculture, Washington, DC.

Bureau of the Census (1932c), Fifteenth Census of the United States: 1930. Outlying Territories and Possessions, Washington, DC.

Bureau of the Census (1942d), Sixteenth Census of the United States: 1940. Reports for Puerto Rico: Census of Agriculture, Washington, D.C.

Camuñas, Manuel, Commissioner of Agriculture and Labor (1917a), Report of the Commissioner of Agriculture and Labor of Porto Rico, 1917. From the Report of the Governor of Porto Rico, 1917, pages 545-562 inclusive, Washington, Government Printing Office. 
Camuñas, Manuel, Commissioner of Agriculture and Labor (1918b), Report of the Commissioner of Agriculture and Labor of Porto Rico, 1918. From the Report of the Governor of Porto Rico, 1918, pages 621-649 inclusive, Washington, Government Printing Office.

Camuñas, Manuel, Commissioner of Agriculture and Labor (1919c), Report of the Commissioner of Agriculture and Labor of Porto Rico, 1919. From the Report of the Governor of Porto Rico, 1919, pages 685-713 inclusive, Washington, Government Printing Office.

Camuñas, Manuel, Commissioner of Agriculture and Labor (1921d), Report of the Commissioner of Agriculture and Labor of Porto Rico, 1921. From the Report of the Governor of Porto Rico, 1921, pages 448-503 inclusive, Washington, Government Printing Office.

Chardón, Carlos, Commissioner of Agriculture and Labor (1926a), Annual Report of the Commissioner of Agriculture and Labor Submitted to the Governor of Porto Rico, 1924-25, San Juan, Bureau of Supplies, Printing, and Transportation.

Chardón, Carlos, Commissioner of Agriculture and Labor (1927b), Informe del Comisionado de Agricultura y Trabajo al Honorable Gobernador de Puerto Rico, 1925-1926, San Juan, Negociado de Materiales, Imprenta y Transporte.

Chardón, Carlos, Commissioner of Agriculture and Labor (1929c), Informe del Comisionado de Agricultura y Trabajo al Honorable Gobernador de Puerto Rico, 1928, San Juan, Negociado de Materiales, Imprenta y Transporte.

Clark, Victor (1930), Porto Rico and Its Problems. Washington, Brookings Institution.

Comisión Para Proteger el Tabaco de Puerto Rico (1930), Informe Anual de la Comisión para Proteger el Tabaco de Puerto Rico (1929-1930), San Juan, Negociado de Materiales, Imprenta y Transporte.

Cook, Melville T., and José I. Otero (1937), "History of the First Quarter of a Century of the Agricultural Experiment Station at Río Piedras, Puerto Rico", Boletín de la Estación Experimental Agrícola, 44.

Crowley, J. T. (1911), "Organization of the Station and Cultivation of Sugar Cane in Porto Rico", Boletín de la Estación Experimental Agrícola 1, p. 6.

Díaz de Acín, Nilsa (1967), La participación de la Estación Experimental de Agricultura de la Universidad de Puerto Rico en el desarrollo agrícola de Puerto Rico, Master's thesis, University of Puerto Rico.

Esteva, Carlos Jr. (1940a), Third Annual Report of the Tobacco Institute of Puerto Rico (1938-1939), San Juan, Bureau of Supplies, Printing, and Transportation.

Esteva, Carlos Jr. (1942b), Annual Reports of the Tobacco Institute of Puerto Rico. Fiscal Years 1939-1940 and 1940-1941, San Juan, Bureau of Supplies, Printing, and Transportation.
Gandía Córdova, Ramón, Acting Commissioner of Agriculture and Labor (1920), Report of the Commissioner of Agriculture and Labor of Porto Rico, 1920. From the Report of the Governor of Porto Rico, 1920, pages 503-566 inclusive, Washington, Government Printing Office.

Insular Experiment Station of Puerto Rico (1917), Annual Report of the Insular Experiment Station of Puerto Rico for the Period from July 1, 1916 to July 1, 1917, San Juan, Bureau of Supplies, Printing, and Transportation.

Insular Experiment Station of Puerto Rico, Department of Agriculture and Labor of Puerto Rico (1919a), Eighth Annual Report of the Insular Experiment Station of the Department of Agriculture and Labor of Puerto Rico for the Period from July 1, 1917 to June 30, 1918, San Juan, Bureau of Supplies, Printing, and Transportation.

Insular Experiment Station of Puerto Rico, Department of Agriculture and Labor of Puerto Rico (1920b), Tenth Annual Report of the Insular Experimental Station of the Department of Agriculture and Labor of Puerto Rico. Fiscal Year 1919-20, San Juan, Bureau of Supplies, Printing, and Transportation.

Insular Experiment Station of Puerto Rico, Department of Agriculture and Labor of Puerto Rico (1921c), Eleventh Annual Report of the Insular Experimental Station of the Department of Agriculture and Labor of Puerto Rico. Fiscal Year 1920-21, San Juan, Bureau of Supplies, Printing, and Transportation.

Insular Experiment Station of Puerto Rico, Department of Agriculture and Labor of Puerto Rico (1922d), Twelfth Annual Report of the Insular Experimental Station of the Department of Agriculture and Labor of Puerto Rico. Fiscal Year 1921-22, San Juan, Bureau of Supplies, Printing, and Transportation.

Insular Experiment Station of Puerto Rico, Department of Agriculture and Labor of Puerto Rico (1924e), Thirteenth Annual Report of the Insular Experimental Station of the Department of Agriculture and Labor of Puerto Rico. Fiscal Year 1922-23, San Juan, Bureau of Supplies, Printing, and Transportation.

Insular Experiment Station of Puerto Rico, Department of Agriculture and Labor of Puerto Rico (1924f), Fourteenth Annual Report of the Insular Experimental Station of the Department of Agriculture and Labor of Puerto Rico. Fiscal Year 1923-24, San Juan, Bureau of Supplies, Printing, and Transportation.

Insular Experiment Station of Puerto Rico, Department of Agriculture and Labor of Puerto Rico (1931g), Annual Report of the Insular Experimental Station of the Department of Agriculture and Labor of Puerto Rico. Fiscal Year 1929-30, San Juan, Bureau of Supplies, Printing, and Transportation.

López Domínguez, Francisco A. (1926a), Fifteenth Annual Report of the Insular Experimental Station of the Department of Agriculture and Labor of Puerto Rico. Fiscal Year 1924-25, San Juan, Bureau of Supplies, Printing, and Transportation.

López Domínguez, Francisco A. (1929b), Seventeenth Annual Report of Director of the Insular Experimental Station of the Department of Agriculture and Labor of Puerto Rico. Fiscal Year 1926-27, San Juan, Bureau of Supplies, Printing, and Transportation. 
Lugo, Wanda I. y José Arroyo Aguilú (2001), “EEA celebra 90 años a la vanguardia de la ciencia y tecnología agrícola", Crónicas Universitarias, 6 (2), pp. 21-26.

McCook, Stuart (2001), "Promoting the 'Practical': Science and Agricultural Modernization in Puerto Rico and Colombia, 19201940", Agricultural History 75 (1), pp. 52-82.

Menéndez Ramos, Rafael (1933a), Comisionado de Agricultura y Comercio. Informe Anual del Comisionado de Agricultura y Comercio correspondiente al año fiscal 1932-1933, San Juan, Departamento de Agricultura y Comercio.

Menéndez Ramos, Rafael (1934b), Comisionado de Agricultura y Comercio, Informe Anual del Comisionado de Agricultura y Comercio correspondiente al año fiscal 1933-1934, San Juan, Negociado de Materiales, Imprenta y Transporte.
Nolla, J.A.B. (1937), First Report of the Tobacco Institute of Puerto Rico. Fiscal Year 1936-37, San Juan, Bureau of Supplies, Printing, and Transportation.

Rodríguez Géigel, A. (1941), Servicio de Extensión Agrícola. Colegio de Agricultura y Artes Mecánicas de la Universidad de Puerto Rico, Informe Anual del Servicio de Extensión Agrícola. Diciembre 1, 1940 a noviembre 30, 1941, Río Piedras, Universidad de Puerto Rico.

Servicio de Extensión Agrícola. Colegio de Agricultura y Artes Mecánicas de la Universidad de Puerto Rico (1938), Sirviendo al Agro. Informe de las actividades del Servicio de Extensión Agrícola de la Universidad de Puerto Rico. December 1, 1937 to November 30, 1938, Río Piedras, Universidad de Puerto Rico.

Tobacco Institute of Puerto Rico (1945), Tobacco Institute Report. Fiscal Years 1941-42, 1942-43, San Juan, Real Hermanos, Inc. 